\title{
Etnomicología de los lacandones de Nahá, Metzabok y Lacanjá-Chansayab, Chiapas, México
}

\author{
Lacandon Ethnomycology of Nahá, Metzabok and Lacanjá-Chansayab, \\ Chiapas, Mexico
}

Felipe Ruan-Soto ${ }^{1 *}$, Mario Domínguez-Gutiérrez ${ }^{2}$, Lilia Pérez-Ramírez ${ }^{3}$, Joaquín Cifuentes ${ }^{3}$

${ }^{1}$ Laboratorio Taller de Procesos Bioculturales, Educación y Sustentabilidad, Instituto de Ciencias Biológicas. Universidad de Ciencias y Artes de Chiapas, Tuxtla Gutiérrez, Chiapas, México, ${ }^{2}$ Instituto de Ecología, Xalapa, Veracruz, México, ${ }^{3}$ Facultad de Ciencias, Universidad Nacional Autónoma de México, Ciudad de México, México.

*Autor a quien se dirige la correspondencia: ruansoto@yahoo.com.mx

Recibido: 25 de enero de 2021 / Aceptado: 6 de abril de 2021

\section{Resumen}

$\mathrm{D}$ esde los albores del s. XX se produjo una cantidad inusitada de proyectos de investigación y publicaciones acerca de los lacandones, grupo originario del sureste mexicano; particularmente destacan aquellos relacionados con el manejo y aprovechamiento de recursos naturales de la Selva. Pese a este vasto esfuerzo de documentación etnográfica, se cuenta con pocos estudios relacionados con el conocimiento que tienen al respecto de los hongos. A través de métodos y técnicas etnográficas y aproximaciones para análisis cuantitativos desarrolladas a lo largo de casi cuatro años de trabajo de campo, el presente estudio muestra algunas de las especies que son reconocidas por este grupo, narrativas relacionadas con el lugar de estos organismos en su mitología, aspectos de su sistemática y nomenclatura, el uso y manejo de ciertas especies, así como un análisis cuantitativo de la similitud de conocimientos etnomicológicos entre las tres comunidades de lacandones donde se trabajó: Nahá, Metzbok y Lacanjá Chansayab. Los Lacandones reconocen y nombran en conjunto 164 especies. Los nombres asignados a los hongos por lo general parten de una descripción morfológica de las características propias de las especies. Del total de especies reconocidas, 37 de ellas tienen algún uso, principalmente como comestibles y medicinales. Si bien la mayoría de las especies no tienen un uso directo, sí tienen una relevancia manifestada en distintos aspectos lingüísticos y de su lugar en la cosmovisión de este grupo. Aparentemente existen diferencias en el conocimiento etnomicológico evaluado a través de la Importancia cultural de las especies y sus prácticas de aprovechamiento entre las poblaciones de lacandones del norte y del sur.

Palabras clave: Hongos comestibles, conocimiento micológico tradicional, etnobiología, patrimonio biocultural, Selva Lacandona

\section{Abstract}

Grom the beginning of the twentieth century a remarkable amount of research projects and publications about the Lacandons, a Maya people from southeast Mexico, were produced; prominent among these were those related to their use and management of natural resources. Despite this massive ethnographic effort, studies focused on Lacandon knowledge of mushrooms remained scarce. Through ethnographic methods and techniques, as well as approaches for quantitative analysis developed over almost four years of fieldwork, this study presents some fungi species recognized by this human group, narratives illustrating their role in Lacandon mythologies, their systematic and nomenclature aspects, and their use and management, as well as a quantitative analysis of the similitude of ethnomycological knowledge between Lacandon communities (Nahá, Metzbok y Lacanjá Chansayab). The Lacandon know and name 164 species. Names assigned to mushrooms generally come from morphological descriptions of the features of a species. From all known species, 37 are used, mainly as food and medicine. There appear to be differences in ethnomycological knowledge between northern and southern Lacandons according to the Cultural Significance of the species and its management.

Keywords: Edible mushrooms, traditional mycological knowledge, ethnobiology, biocultural heritage, Lacandon rainforest 


\section{Introducción}

Hach winik se refiere a la forma en que los lacandones se autodenominan y significa en su lengua "verdaderos hombres". Los lacandones son quizá el grupo originario que se hace más presente por las personas en México y que ha permanecido por más tiempo en el foco de atención para exploradores, investigadores, organismos de gobierno y la sociedad civil desde su "descubrimiento", siendo representados ampliamente en fotografías, grabaciones, notas, por mencionar algunos medios (Trench, 2005).

Aunque el origen de este pueblo resulta un tanto incierto, aparentemente se ha ido conformando a partir de diversos pueblos rebeldes que encontraron en la selva tropical lluviosa del sureste mexicano, un refugio para resguardarse de los embates de reducción del gobierno colonial (De Vos, 1980). Si bien desde el siglo XVIII se dieron algunos contactos a través de intentos de cristianización, sobre todo a los lacandones del sur (Erosa, 2006), las poblaciones de este grupo se mantuvieron relativamente aisladas del contacto con el exterior hasta finales del siglo XIX, cuando comenzó la explotación maderera de la selva (Roblero, 2008).

Este relativo aislamiento y la aparente permanencia de una forma de vida muy antigua, e inclusive lo que en su momento Tozzer caracterizó como los últimos vestigios de la religión del periodo clásico maya (Cano-Contreras et al., 2009), despertó un interés persistente de muchos viajeros e investigadores por estudiar a estos puestos sobrevivientes del clásico maya que no fueron "contaminados por la experiencia colonial" (Trench, 2005, p. 53). Desde la aparición de las primeras etnografías en la primera década del siglo XX (Tozzer, 1982), y después con mucho mayor frecuencia gracias a las incursiones a través de avionetas en la década de los cuarenta del siglo. XX, se produjeron una cantidad inusitada de proyectos de investigación y la publicación de monografías, artículos y tesis. Para la década de los ochentas, se contabilizaban más publicaciones que lacandones (Perera \& Bruce, 1982, en Trench, 2005). Aunado a los trabajos relacionados con aspectos de parentesco, religión, organización social, lingüística, por mencionar algunos, destacan los estudios realizados en torno al manejo y aprovechamiento de recursos naturales (Contreras \& Mariaca, 2016). Uno de los primeros estudios etnobotánicos fue el de Nations y Nigh (1980), al respecto del manejo tradicional de la milpa y los acahuales. A este le siguieron una diversidad de trabajos relacionados con el uso de plantas y animales como alimento y medicina (Cook, 2016; Kashanipour \& McGee, 2004), las tecnologías utilizadas para el manejo de sus ecosistemas (Marion Singer, 1999) y el uso de ciertas especies de plantas en las dinámicas de aprovechamiento y descanso de las parcelas (Levy-Tacher et al., 2002).

Pese a este vasto esfuerzo de documentación etnográfica e histórica, se tienen pocos estudios relacionados con el conocimiento que tienen los lacandones al respecto de los hongos. Los hongos son uno de los grupos de organismos más diversos sobre el planeta, se estima que en el mundo deben existir entre 2.2 y 3.8 millones de especies (Hawksworth \& Lücking, 2017). Solamente para las selvas chiapanecas, se estima deben existir alrededor de 150,000 especies de hongos (Ruan-Soto et al., 2017). Estos organismos pueden tener diferentes modos de vida, como saprobios (se les considera como los principales descomponedores de materia orgánica, permitiendo el reciclaje de nutrimentos y restableciendo sus niveles en los suelos), simbiontes (como las micorrizas, que ayudan a las plantas intercambiando nutrientes y agua por alimento) o parásitos en ocasiones. Además de cumplir estas funciones ecológicas, los hongos también son alimento para muchos insectos y mamíferos (Garibay-Orijel \& García-Medrano, 2006). Todas estas particularidades no han pasado inadvertidas por los grupos humanos y han sido aprovechados a lo largo del tiempo por sus propiedades alimenticias, medicinales y enteógenas, por mencionar algunas (Moreno-Fuentes \& Garibay-Orijel, 2014). Esto ha sido recopilado en diversas publicaciones etnomicológicas a lo largo del mundo (Comandini \& Rinaldi, 2020; Moreno-Fuentes et al., 2001). Recientemente se ha utilizado el concepto de importancia cultural para evaluar cualitativa, pero sobre todo cuantitativamente, la relevancia que pueden tener ciertas especies de hongos sobre otras. (Garibay-Orijel et al., 2007; Montoya et al., 2012). Hunn (1982) definió la importancia cultural de un taxón como el valor del papel que desempeña el organismo dentro de una cultura particular.

En el estado de Chiapas, México, se ha evaluado cuáles son las especies de hongos de mayor importancia cultural para grupos mayas de tierras altas (Ruan-Soto, 2018a; 2020), así como aspectos relacionados con su uso y manejo por pueblos mayas del sureste mexicano y Guatemala (Ruan-Soto \& OrdazVelázquez, 2015). Para el caso de los mayas lacandones, se ha realizado la recopilación de algunos datos etnográficos y taxonómicos respecto de algunas espe- 
cies de hongos macroscópicos (Ruan-Soto et al., 2007; 2017).

El presente estudio señala algunas de las especies que son reconocidas por los lacandones, algunas narrativas relacionadas con el lugar de estos organismos en su mitología, aspectos de su sistemática y nomenclatura, el uso y manejo de ciertas especies, así como un análisis cuantitativo de la similitud de sus conocimientos etnomicológicos entre comunidades.

\section{La Selva Lacandona}

\section{Diversidad biológica de la Selva Lacandona}

La Selva Lacandona se ubica en la porción Este del estado de Chiapas, donde ocupa porciones de los municipios de Ocosingo, Las Margaritas, Marqués de Comillas, Maravilla Tenejapa, Benemérito de las Américas, Palenque, Altamirano y Chilón (Durand et al., 2014). Es una cuenca hidrológica de gran importancia que se extiende por casi $13,000 \mathrm{~km}^{2}$ (Castillo-Campos \& Narave, 1992) y cuyos aportes pertenecen al sistema Grijalva-Usumacinta. Las principales corrientes permanentes de la zona son los ríos Perlas, Jataté, Euseba y Lacantún; entre los cuerpos lacustres permanentes destacan los lagos Ocotal, Metzabok, Lagunas de Lacanjá y Miramar (García-Gil \& Lugo, 1992).

En la región se distinguen tres tipos de formas de relieve: laderas, mesas y depresiones, constituidas por calizas del cretácico superior. La región presenta variaciones altitudinales que van desde los $200 \mathrm{~ms}$ n.m. hasta los 1,500 en la parte norte (Instituto Nacional de Ecología[INE], 2000). Se han reconocido varios tipos de suelos como litosoles, luvisoles y fluvisoles; en general, son suelos ácidos con una capa superficial de materia orgánica y poco profundos (García-Gil \& Lugo, 1992). El clima predominante es cálido húmedo con lluvias abundantes en el verano y parte del otoño, así como una temporada seca corta durante los meses de marzo a mayo; la temperatura media anual es de $25{ }^{\circ} \mathrm{C}$ con una precipitación de 2,300 a 2,600 mm (Levy-Tacher, 2000; Quintana-Ascencio et al., 1990).

El tipo de vegetación que se puede encontrar es mayoritariamente la selva alta perennifolia, con presencia adicional de selva mediana subperennifolia (Miranda, 1952). La mayoría de los árboles tienen troncos rectos y grandes contrafuertes. Entre las especies más frecuentes están: Terminalia amazonica (canshán), Ceiba pentandra (ceiba), Swietenia ma- crophylla (caoba), Brosimum alicastrum (ramón), por mencionar algunas (Castillo-Campos \& Nareve, 1992; Levy-Tacher, 2000; Pennington \& Sarukhan, 1998). En las selvas medianas destacan especies como Cedrela odorata (cedro) y Bursera simaruba (chacá) (Castillo-Campos \& Nareve, 1992). En Nahá también se encuentra bosque mesófilo con presencia de Podocarpus matudai, Quercus corrugata y Q. skinneri, así como reducidos bosques de coníferas en los alrededores de las lagunas Ocotalito y Nahá con presencia de Pinus tenuifolia y Oreopanax sp. Metzabok, además contiene bosque espinoso dominada casi exclusivamente por palo de tinto (Haematoxylum campechianum) (Comisión Nacional de Áreas Naturales Protegidas [CONANP], 2006a, 2006b). Sin embargo, debido en gran medida a la actividad humana, las selvas húmedas han ido modificándose, transformándose en pastizales y acahuales en distintos grados de sucesión; reduciendo críticamente las comunidades de selva madura (Durán, 1999).

Lacanjá-Chansayab cuenta con una extensión de 4,357 ha y se ubica geográficamente entre los $16^{\circ} 46^{\prime} 08^{\prime \prime}$ latitud norte y $91^{\circ} 08^{\prime} 12^{\prime \prime}$ longitud oeste, y alrededor de $350 \mathrm{~ms}$ n.m. en el municipio de Ocosingo, Chiapas. Nahá cuenta con una superficie de 3,847 ha y se ubica entre los $16^{\circ} 56^{\prime} 41^{\prime \prime}$ y los $17^{\circ} 00^{\prime} 42^{\prime \prime}$ de latitud norte y entre los $91^{\circ} 32^{\prime} 52^{\prime \prime}$ de longitud oeste. Por su parte, Metzabok cuenta con una superficie de 3,368 ha y se localiza entre los $17^{\circ} 08^{\prime} 36^{\prime \prime}$ y $17^{\circ} 04^{\prime} 53^{\prime \prime}$ de latitud norte y entre los $91^{\circ} 34^{\prime} 42^{\prime \prime}$ y $91^{\circ} 40^{\prime} 09^{\prime \prime}$ de longitud oeste (CONANP, 2006a, 2006b).

Los lacandones, su lengua y sus mitos

Pese a la gran cantidad de estudios y revisiones realizadas (Boremanse, 1978; Bruce 1976; De Vos, 1980; Villa-Rojas, 1985), el origen del pueblo lacandón es un poco incierto. Desde el siglo XVI, las selvas lluviosas de lo que hoy es el sureste de México y norte de Guatemala, dieron refugio a poblaciones que intentaban escapar de los intentos de reducción, reubicación y control del gobierno colonial (De Vos, 1980). De acuerdo con Boremanse (1978) el término 'lacandón' se utilizó para referirse a esas poblaciones de indígenas rebeldes que encontraron refugio en los territorios que hoy conocemos como la selva chiapaneca, el Petén guatemalteco, Belice y la parte sur de la Península de Yucatán. En este sentido, los lacandones actuales, pueden haberse originado de grupos étnicos distintos, que con el tiempo fueron fusionando sus dis- 
tintas tradiciones generando una cultura más homogénea (Marion Singer, 1999).

La lengua que se habla es el maya lacandón, la cual está cercanamente relacionada con el maya peninsular, pertenecientes ambas junto al itzaj y el mopan a la rama yukateka de las lenguas mayas (England, 1996). El maya lacandón tiene dos variantes dialectales, la del norte y la del sur, inteligibles entre ellas (Cano-Contreras et al., 2009).

El relativo aislamiento de los lacandones, hasta prácticamente el siglo XX permitió que su religiosidad y sus mitos de origen se mantuvieran escasamente influidos por el cristianismo.

Los mitos del origen de los lacandones narran cómo se suscitaron los designios divinos que permitieron la creación de la selva, de todo lo que ella contiene y de los mismos lacandones. Estos mitos aquí descritos son extraídos de diferentes textos que abordan aspectos de la cosmovisión lacandona como Boremanse,1978; Bruce et al.,1971; Cano-Contreras et al., 2009, Marion Singer, 2000; Tozzer,1982. El primer dios fue $K^{\prime}$ akoch, quien creo el sol y la luna. Después a partir de una flor de tsaknikté, nacieron tres dioses: Sukunkyum, Äkyatho y Hachäkyum. Hachäkyum 'nuestro verdadero señor', es el dios más importante, ya que fue el que creo la selva llenándola de plantas y animales y le asignó a cada uno un lugar donde vivir. También utilizó tierra y madera podrida para crear a Kisin al anochecer, el cual fue enviado a vivir y reinar junto con Sukunkyum el inframundo. Después creo a los lacandones para que lo venerasen a partir de arcilla, arena y maíz, que puso en sus bocas a manera de dientes. Una vez realizado todo, los dioses dejaron la tierra y se fueron a vivir al cielo, dejando a los hach winik con la encomienda de cuidar la selva.

Las comunidades lacandonas y sus estrategias de aprovechamiento de la selva

Aunque antiguamente los lacandones habitaban en parajes dispersos agrupados en pequeñas poblaciones con familias extensas, hoy en día los lacandones se concentran en cinco poblaciones principales: Naha y Metzabok en el norte; y Lacanja-Chansayab, San Javier y Betel en el sur (Cano-Contreras et al., 2009) (Figura 1).

Los lacandones nunca han tenido una población muy alta (Erosa, 2006). Según datos del Instituto Nacional de Estadística y Geografía (INEGI, 2010) la población total apenas alcanza 926 habitantes.
Para los lacandones, la selva siempre ha sido un reservorio de recursos naturales que les ha permitido satisfacer todas sus necesidades a lo largo del tiempo (Erosa, 2006). Esto basado en un aprovechamiento integral del medio, aprovechando organismos y elementos de diferentes espacios o agroecosistemas como la milpa, el acahual, el huerto, la selva, y los ambientes acuáticos (ríos y lagunas) (Contreras \& Mariaca, 2016).

Nations y Nigh (1980) documentaron cómo las milpas y los acahuales, basados en el sistema de roza-tumba-quema, contienen una riqueza de más de 50 especies de plantas cultivadas y manejadas, además de un eficiente rendimiento y una estrategia de manejo que permite una regeneración rápida de los espacios transformados siguiendo las propias fases de sucesión ecológica de la selva, fomentando la presencia de ciertas especies (Levy-Tacher et al., 2002). Asimismo, la selva provee diferentes especies de mamíferos, reptiles, aves, moluscos y peces que son cazados o pescados (Baer \& Merrifield, 1981; March, 1998) y algunas plantas silvestres que se recolectan para su consumo (Contreras \& Mariaca, 2016).

\section{Figura 1}

Localización de Nahá, Metzabok y Lacanjá-Chansayab, Chiapas, México.

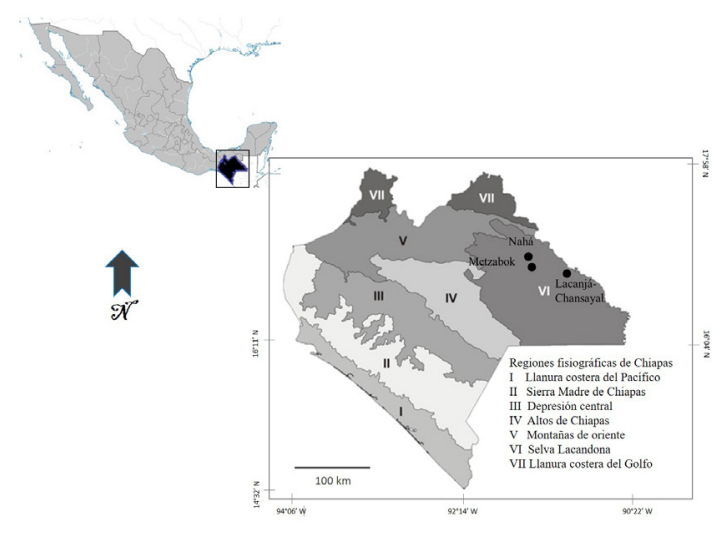




\section{Métodos}

Antes de comenzar el trabajo de campo, se solicitó consentimiento previo, libre e informado, a las autoridades políticas, tradicionales y ambientales (como la Comisión Nacional de Áreas Naturales Protegidas) de las tres comunidades lacandonas donde se llevó a cabo el trabajo para poder realizar las entrevistas a los pobladores que así lo quisieran, para realizar recolectas de hongos y para poder publicar los resultados de esta investigación. Todo el trabajo se llevó a cabo siguiendo los lineamientos del Código de Ética para la investigación, la investigación-acción y la colaboración etnocientífica en América Latina. Versión Dos (Cano-Contreras et al., 2015).

Del 2007 al 2012 se realizaron entrevistas no estructuradas y semiestructuradas (Bernard, 2006) a lacandones de las tres comunidades que quisieran colaborar con la investigación y que fueran reconocidos hacia el interior como poseedores de conocimientos etnoecológicos sobre la selva. Estas personas fueron seleccionadas a través de un muestreo teórico mediante la herramienta de la bola de nieve (Sandoval, 2002), haciendo un total de 28 entrevistados. Las entrevistas fueron realizadas en español con ayuda de traductores espontáneos de la misma comunidad, las cuales giraron en torno a temas relacionados con la taxonomía y clasificación lacandona de los hongos, conocimientos etnoecológicos, usos y narrativas al respecto de su origen.

Asimismo, se realizó un ejercicio de aproximación cuantitativa para evaluar la importancia cultural de los hongos de acuerdo lo propuesto por Hunn (1982). Se realizaron entrevistas estructuradas a 35 lacandones elegidos al azar en las tres comunidades, todos mayores de 18 años (en algunos casos, la selección aleatoria coincidió con los elegidos para el muestreo teórico), las cuales consistían en un listado libre donde se solicitaba que cada entrevistado enunciara los nombres de los hongos que conociera, así como un ejercicio de corroboración taxonómica para saber la identidad taxonómica del etnotaxón mencionado mediante un catálogo fotográfico con los morfotipos más frecuentes encontrados. Este catálogo se construyó de acuerdo con lo sugerido por Thomas y colaboradores (2007) respecto de la proporción, tamaño y definición de la imagen. Para el análisis de esta información, se utilizó la frecuencia de mención como el indicador de la importancia cultural, en este sentido, el etnotaxón más mencionado en las entrevistas fue considerado el más importante (Montoya et al., 2012; Weller \& Romney, 1988). Para explorar las diferencias de estos datos entre los sitios de estudio con base en la frecuencia de mención relativa de las especies se calculó una matriz de distancia por el método de distancia taxonómica promedio. Con estos valores se realizaron análisis de cluster por el método UPGMA (Unweighted PairGroup Method using Arithmetic averages) con ayuda del programa NTSYS versión $2.11 \mathrm{x}$ para computadora personal (Rohlf, 2005).

Para la identificación de las especies fúngicas referidas en las entrevistas se realizaron recorridos etnomicológicos (Ruan-Soto, Ramírez-Terrazo et al., 2020) donde se recolectaban los ejemplares. Estos fueron descritos en cuanto a sus características macroscópicas, fotografiados y herborizados de acuerdo con lo propuesto por Cifuentes y colaboradores (1986) y posteriormente revisados microscópicamente según lo propuesto por Largent y colaboradores (1977). Para determinar los ejemplares se utilizaron diferentes claves taxonómicas especializadas, descripciones y guías de campo.

\section{Resultados y discusión}

\section{El origen de los hongos}

Dentro de las narrativas lacandonas se mencionan algunos relatos que dan cuenta de cómo se piensa y se explica el origen de los hongos en el mundo. Para los lacandones, Hacha 'kyum, quien es la deidad principal, creó todas las montañas, las lagunas, todos los animales, las plantas y todo lo necesario para que los Hach winik o verdaderos hombres pudieran vivir. Asimismo, se cuenta que cuando Hacha 'kyum, cansado, se sentó a contemplar toda su creación; como tenía hambre, tomó unos trozos de madera del tronco del árbol donde se había sentado y los comió. Los pedazos que se le cayeron sobre los troncos dieron origen a los kuxum che' u hongos de los palos, en tanto que los pedazos que cayeron sobre el suelo dieron origen a los kuxum lu'um u hongos de la tierra. Las narrativas mencionan que los lacandones, quienes observaban todo este episodio, tuvieron antojo de comer los manjares que saboreaba su padre e inmediatamente se atrevieron a pedirle que compartiera con ellos lo que estaba comiendo. Hacha'kyum les dijo que esos hongos no los podían comer porque eran la comida de él y el Kisin, eran comida de dioses. Asimismo, les dijo que los hongos que había creado eran las herra- 
mientas y utensilios que usaría Kisin cuando caminara por la Selva. Sin embargo, como vio la decepción en sus hijos, les dijo que no se apenaran, que purificaría algunos hongos para que los lacandones pudieran recolectar y comer. Así creo el kayoch, el xikin, el lo'ro y otros hongos más para que pudieran disfrutar de estos bienes. El resto de los hongos, quedarían reservados para su uso de los Dioses.

Al momento de la creación de la tierra, de los ríos, de los árboles, las plantas y todo lo que miramos, Hacha'kyum agarró un pedazo de madera y la transformó en comida para él, para ver todo lo que hizo mientras comía, el mundo que había creado, pero lo dejó, no lo terminó y así fue como quedo en la tierra el kuxum lu'um y en el tronco quedó el Kuxum che'. La comida de los dioses, pero él también nos purificó unos para nosotros como el Kayoch...Hacha'kyum purificó algunos hongos para que tuviéramos que comer, porque se nos antojaba mucho, parecían panes sabrosos horneados en la selva, pero no podíamos comerlos porque eran de Kisin, por eso se purificaron y ahora salen en nuestras milpas, en nuestros caminos, a nuestro paso (A. Chan Kin, comunicación personal, Nahá, julio de 2007).

Cuando Hacha'kyum creó el mundo, contempló su creación sentado sobre un tronco y de ahí creó el Kuxum che', porque tenía hambre, pero dejó un poco y desde ahí empiezan a salir en los árboles caídos y también algunos vivos y ese es el pan de los Dioses (K. García, comunicación personal, Nahá, agosto de 2008).

Kisin sale de las cuevas en las noches, y está metido en la selva y nos damos cuenta donde estuvo, porque deja su comida, su wah kisin, y el plato donde lo comió su tortilla...Kisin también tiene sus herramientas y están en la selva porque también es su hogar, es como nosotros, tiene su banca, su sombrero, su escoba, su cepillo, también le gusta estar arreglado y cuando se peina deja sus pelos en el monte (M. Koh, comunicación personal, Nahá, agosto de 2008).

Estas narrativas, independientemente del valor que tienen como parte de la tradición oral del pueblo maya lacandón, dan muchas pistas acerca del porqué de diversos aspectos de la etnomicología maya como su sistema de clasificación y nomenclatura de las especies fúngicas, así como de las especies utilizadas como alimento. López Austin define la cosmovisión como "el conjunto estructurado de los diversos sistemas ideológicos con los que el grupo social, en un momento histórico, pretende aprehender el universo, engloba todos los sistemas, los ordena y los ubica" (López Austin, 1990, p. 20). El constructo socializado implica distintas formas de explicar el mundo y la presencia de las cosas que hay en él (Cano-Contreras et al., 2009). Además, los relatos dejan ver ciertos aspectos de la cosmovisión de los mayas lacandones y el lugar que tienen los hongos en el orden de su universo, lo cual definitivamente marca una pauta que dirige la forma de enunciar las diferentes especies de hongos que existen en la selva y, sobre todo, la manera de relacionarse con ellos, como se verá a continuación tanto en términos de su lengua como en términos de sus usos.

\section{Especies de hongos reconocidas por los lacandones} y su sistemática

Los Lacandones de estas tres comunidades reconocen y nombran en conjunto 164 especies (Tabla 1). Estas especies se agrupan en 49 familias taxonómicas y 18 órdenes. El 14\% de las especies corresponden al phyllum Ascomycota, en tanto que el $86 \%$ corresponden a Basidiomycota. Destaca el alto número de especies reconocidas de la familia Xylariaceae (12) y Sarcoscyphaceae (7). Respecto a sus sustratos, 103 especies son lignícolas, 49 terrícolas, 11 humícolas, y una se encontró sobre la mazorca del maíz como parásito.

Antiguamente, la literatura señalaba de manera persistente que en las tierras bajas tropicales la gente no tiene mucho conocimiento de las especies fúngicas o inclusive que estas no tienen un papel tan relevante en los modos de vida de los campesinos, a diferencia de lo observado en tierras altas mesoamericanas (Goés-Neto \& Bandeira, 2003; Guzmán, 1987; Mapes et al., 2002). Sin embargo, estas aseveraciones se han ido matizando por distintas investigaciones que han demostrado la importancia que tienen los hongos en tierras bajas tropicales de América del Sur (VascoPalacios et al., 2008; Zent et al., 2004), Asia (Chang \& Lee, 2004), África (Codija \& Yorou, 2014) y por supuesto de Mesoamérica (Hernández-Sánchez, 2007; Jiménez, 2017; Ruan-Soto et al., 2004). Incluso es de llamar la atención en el número de especies que reconocen y nombran los lacandones comparado con otros grupos mayas de Chiapas (Ruan-Soto, 2018b).

El que la mayoría de las especies reconocidas por los lacandones, cerca del $63 \%$, son de sustrato lignícola puede deberse, en principio, a que en las selvas estos hongos son los más abundantes debido a la gran cantidad de ramas y materia orgánica en el sotobosque que puede ser utilizado por estos organismos, situación distinta en los bosques templados donde las 
especies terrícolas y/o micorrizógenas son mucho más abundantes (Guzmán-Dávalos \& Guzmán, 1979; Ruan-Soto, Cifuentes et al., 2020).

Berlin (1992) define la existencia de principios que reflejan patrones generales relacionados a la clasificación de la biota. De manera general propone que en todos los grupos humanos es posible identificar grupos de organismos (taxones) que se reconocen en el lenguaje y que se basan en gradientes de mayor o menor exclusión; dichos taxones se agrupan en categorías etnobiológicas y se van a distribuir entre rangos mutuamente excluyentes (reino, forma de vida, intermedio, genérico, específico, variedad) donde taxones del mismo rango comparten grados similares de variación al interior. De acuerdo con lo que se ha observado, la sistemática lacandona parece adecuarse a esta propuesta teórica.

Los lacandones reconocen a los hongos como una entidad totalmente diferente a las plantas y a los animales, denominándolos como kuxum, el cual podría considerarse como un rango de reino según lo propuesto por Berlin (1992). De la misma manera, otros grupos humanos también colocan a los hongos en una categoría separada de plantas y animales como los Txucarrama de la Amazonía brasileña (Cardoso et al., 2010) o los purépechas del occidente de México (Mapes et al., 1981).

Los kuxum se distinguen de los demás organismos por su capacidad intrínseca de pudrir y transformar la materia orgánica. Este término engloba a todos los hongos, tanto los macromicetos como los micromicetos. Este proceso ecológico es visible para los Lacandones e inclusive está perfectamente asociado al flujo de nutrientes y sucesión característicos de la selva y donde los hongos cumplen un papel fundamental. Incluso en el proceso de elaboración del pozol agrio (bebida tradicional hecha a base de maíz fermentado), los lacandones reconocen que el moho que participa en el proceso también es kuxum.

El kayoch sale de los palos que están tirados en la milpa, eso palos ahí se dejan por que el kayoch es que los pudre, así la tierra va a ser más rica y va a haber más maíz, porque si hay más hongos será mejor la milpa (M. Castellanos Chan Kin, comunicación personal, Lacanjá-Chansayab, octubre de 2010).

Kuxum che', kuxum lu'um, es el moho que sale cuando dejas las tortillas en la noche, eso mismo pasa en la tierra, en los palos caídos y animales muertos se convierte y sale kuxum che' y kuxum lu'um" (N. Paniagua, comunicación personal, Nahá, agosto de 2008).
Al interior del grupo de los Kuxum, los lacandones distinguen dos taxones los Kuxum che' y los kuxum lu'um, los primeros para referir a los hongos lignícolas y los segundos para referir a los hongos terrícolas, aspecto que sin duda hace referencia al mito de origen de los hongos descrito anteriormente. Estos dos taxones pueden corresponder al rango - forma de vida - los cuales de acuerdo con Berlin (1992) designan un número de morfotipos de organismos que comparten patrones generales de hábito, sustrato y forma del cuerpo.

Como puede verse en la Tabla 1, los nombres asignados a los hongos por lo general parten de una descripción morfológica de las características propias de las especies como su color (chäk 'rojo', sak 'blanco'), con el parecido que guardan las especies con objetos de la vida cotidiana ( $P$ 'ook 'sombrero', toop' 'flor', misib 'escoba'), con partes del cuerpo (yak' 'lengua', xikin 'oreja'); asociados a ciertos animales (Bayo ch 'bolsa de tlacuache', Choch-el wakäx 'panza de ganado', ch'o' 'ratón'). Esto coincide con lo planteado por Berlin (1992) quien menciona que la nomenclatura etnobiológica alude generalmente a rasgos morfológicos asociados a su referente biológico. Sin embargo, resalta el mito de origen de los hongos descrito anteriormente, que marca la pauta para nombrar a los hongos que puede aprovecharse de manera alimentaria, y aquellos hongos relacionados con entidades divinas y reservados para dicha condición. En este sentido, muchos de los hongos que son aprovechados, sobre todo como alimento, tienen un nombre propio no analizable, a diferencia de los que no son aprovechados, los cuales tienen un nombre relacionado con su morfología general y su asociación a Kisin, como por ejemplo halal kisin 'flecha del kisin' (Xylaria longipes), p'ook kisin 'sombrero del kisin (Agaricus sp., Lepiota sp. Hygrocybe spp., entre otras), misib kisin 'escoba del kisin' (Pterula plumosoides) o wah kisin 'tortilla del kisin' (Phellinus gilvus, Ganoderma lobatum, entre otras).

Muchos etnotaxones que podemos encontrar son politípicos, es decir, los lacandones dan un mismo nombre local para muchas especies de hongos, hay una subdiferenciación. Tal es el caso de los Baak-el kisin 'hueso del kisin' que incluye diferentes especies como Clavulina sp., Clavulinopsis sp., Ramariopsis sp. o Xylaria spp., todas ellas de forma elongada y/o ramificada. La presencia de taxones politípicos es un fenómeno ampliamente presente en diferentes pueblos en Mesoamérica y la Amazonía (Cardoso et al., 2010; 
Ruan-Soto, 2018b). Entre los lacandones se pueden encontrar tanto nombres binomiales como primarios o monomiales, tal como lo describe Berlin (1992). Ejemplo de los primeros tenemos al sak lor'ro 'lo'ro blanco'(Phaeotremella foliacea) o el chäk xikin 'oreja roja' (Cookeina aff. venezuelae) que se componen de una parte que funciona como categórico genérico y un adjetivo calificativo describiendo en este caso su color. En el caso de los segundos tenemos el kayoch (Pleurotus djamor), o el lo'ro (Auricularia spp.), nombres propios no analizables que, de acuerdo con lo propuesto por Turner (1988), pueden ser también los de mayor importancia cultural para las personas en cuestión.

Tabla 1

Especies de hongos macroscópicos reconocidos por los mayas lacandones de Nahá, Metzabok y Lacanjá Chansayab, Chiapas, México

\begin{tabular}{|c|c|c|c|}
\hline Especie & $\begin{array}{l}\text { Nombre lacandón (traducción al } \\
\text { español cuando fue posible) }\end{array}$ & Uso & Sustrato \\
\hline Agaricus moelleri Wasser & Ne nanech & $\mathrm{Ve}$ & $\mathrm{Te}$ \\
\hline Agaricus sp.1 & P'ook kisin (sombrero del kisin) & $\mathrm{Su}$ & $\mathrm{Te}$ \\
\hline Agaricus sp. 2 & Muruch, Much' & Co & $\mathrm{Te}$ \\
\hline Agaricus sp.2 & Muruch & $\mathrm{Co}$ & $\mathrm{Te}$ \\
\hline Agaricus sp.3 & Much' & Co & $\mathrm{Te}$ \\
\hline Agaricus sp.4 & Ushama chil kin & $\mathrm{Ve}$ & $\mathrm{Te}$ \\
\hline Amanita complex. caesarea (Scop.) Pers. & Much' & Co & $\mathrm{Te}$ \\
\hline Amauroderma preussii (Henn.) Steyaert & Upo'ok ti ja, moi'tsimin & $\mathrm{Ve}$ & $\mathrm{Li}$ \\
\hline Astraeus hygrometricus (Pers.) Morgan & Yak' (lengua) & $\mathrm{Su}$ & $\mathrm{Te}$ \\
\hline Aureoboletus auriporus (Peck) Pouzar & Ch'o'wah (tortilla del ratón) & $\mathrm{Su}$ & $\mathrm{Te}$ \\
\hline Auricularia delicata (Mont.) Henn. & $\begin{array}{l}\text { Lo'ro, Choch-el wakäx lo'ro (lo'ro de } \\
\text { panza de ganado) }\end{array}$ & Co & $\mathrm{Li}$ \\
\hline Auricularia fuscosuccinea (Mont.) Henn. & Lo'ro & Co & $\mathrm{Li}$ \\
\hline Auricularia mesenterica (Dicks.) Pers. & Kuxum che'(hongo de árbol) & $\mathrm{Su}$ & $\mathrm{Li}$ \\
\hline $\begin{array}{l}\text { Auricularia nigricans (Sw.) Birkebak, Looney \& Sán- } \\
\text { chez-García }\end{array}$ & Lo'ro & Co & $\mathrm{Li}$ \\
\hline Boletellus ananas (M.A. Curtis) Murrill & Bay och mu'go (bolsa de tlacuache) & $\mathrm{Su}$ & $\mathrm{Te}$ \\
\hline Boletinellus exiguus (Singer \& Digilio) Watling & Tam-en wakäx (hígado de vaca) & $\mathrm{Su}$ & $\mathrm{Li}$ \\
\hline Boletus pulverulentus $f$. pulverulentus Opat. & Wah kisin (tortilla del kisin) & $\mathrm{Su}$ & $\mathrm{Te}$ \\
\hline Cantharellus sp. & Kuxum lu'um (hongo de la tierra) & $\mathrm{Su}$ & $\mathrm{Te}$ \\
\hline Cerrena caperata (Berk.) Zmitr. & Wah kisin (tortilla del kisin) & $\mathrm{Su}$ & $\mathrm{Li}$ \\
\hline Clavaria cf. subacuta S. Ito \& S. Imai & Baak-el kisin (hueso del kisin) & $\mathrm{Su}$ & $\mathrm{Li}$ \\
\hline Clavaria sp. & Baak-el kisin (hueso del kisin) & $\mathrm{Su}$ & $\mathrm{Te}$ \\
\hline Clavulina sp.1 & Baak-el kisin (hueso del kisin) & $\mathrm{Su}$ & $\mathrm{Te}$ \\
\hline Clavulina sp.2 & Baak-el kisin (hueso del kisin) & $\mathrm{Su}$ & $\mathrm{Hu}$ \\
\hline Clavulinopsis aurantiocinnabarina (Schwein.) Corner & Baak-el kisin (hueso del kisin) & $\mathrm{Su}$ & $\mathrm{Te}$ \\
\hline Collybia sp.1 & $\begin{array}{l}\text { Bay och su compañero (bolsa de tla- } \\
\text { cuache) }\end{array}$ & $\mathrm{Su}$ & $\mathrm{Te}$ \\
\hline Collybia sp.2 & Usakp'ook (sombrero blanco) & $\mathrm{Su}$ & $\mathrm{Te}$ \\
\hline Coltricia cinnamomea (Jacq.) Murrill & Chuche wah & $\mathrm{Su}$ & $\mathrm{Li}$ \\
\hline Cookeina colensoi (Berk.) Seaver & Chäk chaach (manojos rojos) & $\mathrm{Su}$ & $\mathrm{Li}$ \\
\hline
\end{tabular}


Cookeina speciosa (Fr.) Dennis

Cookeina tricholoma (Mont.) Kuntze

Cookeina aff. venezuelae (Berk. \& M.A. Curtis) Le Gal

Cookeina venezuelae (Berk. \& M.A. Curtis) Le Gal

Coprinellus truncorum (Scop.) Redhead, Vilgalys \& Moncalvo

Cordyceps militaris (L.) Link

Cortinarius subsect. Phlegmacium sp.

Cotylidia aurantiaca (Pat.) A.L. Welden

Cotylidia diaphana (Schwein.) Lentz

Crepidotus sect. Nyssicolae sp.

Cyathus colensoi Berk.

Dacryopinax elegans (Berk. \& M.A. Curtis) G.W.

Martin

Daedalea quercina (L.) Pers.

Daldinia concentrica (Bolton) Ces. \& De Not.

Daldinia eschscholtzii (Ehrenb.) Rehm.

Earliella scabrosa (Pers.) Gilb. \& Ryvarden

Entoloma sp.1

Entoloma sp.2

Entoloma sp.3

Favolus tenuiculus P. Beauv.

Filoboletus gracilis (Klotzsch ex Berk.) Singer

Flabellophora parva Corner

Fomes fasciatus (Sw.) Cooke

Fuscopostia fragilis (Fr.) B.K. Cui, L.L. Shen \& Y.C.

Dai, in Shen, Wang, Zhou, Xing, Cui \& Dai

Ganoderma australe (Fr.) Pat.

Ganoderma complex. lucidum (Curtis) P. Karst.

Ganoderma lobatum (Schwein.) G.F. Atk.

Ganoderma resinaceum Boud.

Geastrum saccatum Fr.

Geastrum schweinitzii (Berk. \& M.A. Curtis) Zeller

Gomphus sp.

Gymnopus montagnei (Berk.) Redhead

Gymnopus sp.

Hexagonia aff. glabra (P. Beauv.) Ryvarden

Hydnodon thelephorus (Lév.) Banker

Hydnopolyporus palmatus (Hook.) O. Fidalgo

Hydnoporia tabacina (Sowerby) Spirin, Miettinen \&

K.H. Larss.

Hygrocybe conica (Schaeff.) P. Kumm.

Hygrocybe miniata (Fr.) P. Kumm.

Hygrocybe sp.1

Hygrocybe sp. 2

Hygrocybe sp.3
Chäk chaach (manojos rojos)

Chäk xikin (oreja roja) chäk chaach

(manojos rojos)

Chäk xikin (oreja roja)

Chäk chaach (manojos rojos)

Much' su compañero

Baak-el kisin (hueso del kisin)

Yak' much' (much' de lengua)

Upakar kisin, Wah kisin (tortilla del

kisin)

Upakar kisin

Chäk wah (tortilla roja)

Xikin chäk ach

Kuxum che' (hongo de árbol), Lo'ro su compañero

Wah kisin (tortilla del kisin) $\quad \mathrm{Su} \quad \mathrm{Li}$

wuch kuxum

Boox kisin (cáscara del kisin))

Pim wah kisin (tortilla gruesa del kisin)

Yok

Chäk wah (tortilla roja)

P'ook kisin (sombrero del kisin)

Kayoch, Xikin wakax,

P'ook kisin (sombrero del kisin)

Wah kisin (tortilla del kisin)

Wah kisin (tortilla del kisin)

Uk yoch sak pet kisin

Wah kisin (tortilla del kisin)

Wah kisin (tortilla del kisin)

Wah kisin (tortilla del kisin)

Wah kisin (tortilla del kisin)

Uchur kisin, Kuxum lu'um (hongo de tierra)

Yak' (lengua)

Uyus, Lo'le kisin

Lek

Ik'al tsu tsuy

Wah kisin (tortilla del kisin)

Yax wah kisin (tortilla verde del kisin)

Kuxum che' (hongo de árbol)

Kuxum che' (hongo de árbol)

P'ook kisin (sombrero del kisin)

P'ook kisin (sombrero del kisin)

Chäk kuxum (hongo rojo)

P'ook kisin, Chäk lu'um (tierra roja),

Chuk much'

Chäk much' (much' rojo)
Co,

$\mathrm{Lu}$

$\mathrm{Su}$

$\mathrm{Su}$

$\mathrm{Li}$

$\mathrm{Lu}-\mathrm{Li}$

Ve Te

$\mathrm{Su} \quad \mathrm{Te}$

Ve Te

$\mathrm{Su} \quad \mathrm{Li}$

$\mathrm{Su} \quad \mathrm{Li}$

$\mathrm{Su} \quad$ LI

Ag $\mathrm{Li}$

$\mathrm{Su} \quad \mathrm{Li}$

$\mathrm{Su} \quad \mathrm{Li}$

$\mathrm{Su} \quad \mathrm{Li}$

su $\quad \mathrm{Li}$

Ve $\quad$ Te

$\mathrm{Su} \quad \mathrm{Te}$

$\mathrm{Su} \quad \mathrm{Te}$

Co $\quad \mathrm{Li}$

$\mathrm{Lu} \quad \mathrm{Li}$

$\mathrm{Su} \quad \mathrm{Li}$

$\mathrm{Su} \quad \mathrm{Li}$

Em Li

$\mathrm{Me} \quad \mathrm{Li}$

$\mathrm{Me} \quad \mathrm{Li}$

$\mathrm{Su} \quad \mathrm{Li}$

$\mathrm{Su} \quad \mathrm{Li}$

$\mathrm{Me} \quad \mathrm{Te}$

$\mathrm{Su} \quad \mathrm{Li}$

Ve Te

$\mathrm{Lu} \quad \mathrm{Li}$

$\mathrm{Su} \quad \mathrm{Li}$

$\mathrm{Su} \quad \mathrm{Li}$

$\mathrm{Su} \quad \mathrm{Li}$

$\mathrm{Su} \quad \mathrm{Li}$

$\mathrm{Su} \quad \mathrm{Li}$

$\mathrm{Su} \quad \mathrm{Te}$

$\mathrm{Su} \quad \mathrm{Te}$

$\mathrm{Su} \quad \mathrm{Li}$

$\mathrm{Su} \quad \mathrm{Li}$

$\mathrm{Su} \quad \mathrm{Hu}$ 
Hygrocybe sp.4

Hygrocybe sp.5

Hygrophoropsis aurantiaca (Wulfen) Maire

Hygrophorus sp.

Lachnocladium sp.

Lactarius chrysorrheus Fr.

Lactarius indigo (Schwein.) Fr.

Lactarius yazooensis Hesler \& A.H. Sm.

Laetiporus sulphureus (Bull.) Murrill

Lentinula boryana (Berk. \& Mont.) Pegler

Lentinus aff. tricholoma (Mont.) Zmitr.

Lentinus tricholoma (Mont.) Zmitr.

Lentinus berteroi (Fr.) Fr.

Lepiota $\mathrm{sp} .1$

Lepiota sp. 2

Leucocoprinus cepistipes (Sowerby) Pat.

Lycoperdon perlatum Pers.

Lycoperdon sp.

Lycoperdon umbrinum Pers.

Macrolepiota excoriata (Schaeff.) Wasser

Marasmiellus sp. 1

Marasmiellus sp.2

Marasmius sp.

Marasmius cladophyllus Berk.

Microporellus obovatus (Jungh.) Ryvarden

Mycena complex. pura (Pers.) P. Kumm.

Mycena sp.1

Mycena sp.2

Omphalina sp.1

Omphalina sp. 2

Oudemansiella canarii (Jungh.) Höhn.

Panaeolus sp.

Panus neostrigosus Drechsler-Santos \& Wartchow

Perenniporia sp.

Phaeoclavulina cokeri (R.H. Petersen) Giachini

Phaeoclavulina zippelii (Lév.) Overeem

Phaeotrametes decipiens (Berk.) J.E. Wright

Phaeotremella fimbriata (Pers.) Spirin \& V. Malysheva

Phaeotremella foliacea (Pers.) Wedin, J.C. Zamora \& Millanes

Phallus indusiatus Vent.

Phellinus fastuosus (Lév.) Ryvarden

Phellinus gilvus (Schwein.) Pat.

Phellodon melaleucus (Sw. ex Fr.) P. Karst.

Phillipsia domingensis (Berk.) Berk. ex Denison

Phillipsia sp.

Phylacia poculiformis (Mont.) Mont.
P'ook kisin (sombrero del kisin)

$\mathrm{Su}$

Chäk kuxum (hongo rojo)

Chäk xikin (oreja roja)

P'ook kisin (sombrero del kisin)

Baak-el kisin (hueso del kisin)

Kuxum lu'um (hongo de la tierra)

Wah kisin Yax (tortilla verde del kisin),

Yax much' (much' verde)

Yoch

Wah kisin (tortilla del kisin)

Yoch wah kisin (tortilla del kisin)

Toop' kuxum (hongo de flor)

P'ook kisin (sombrero del kisin)

$P^{\prime}$ 'ook kisin (sombrero del kisin)

$P$ 'ook kisin (sombrero del kisin)

Säk pet (cosa redonda y blanca)

Se crok

Kak kisin (cuarto del kisin)

Ye'er kisin (huevo del kisin)

Uchur kisin

Bu'jo much'

Kuxum che' (hongo de árbol)

$P$ 'ook kisin (sombrero del kisin)

$P$ 'ook kisin (sombrero del kisin)

P'ook kisin (sombrero del kisin)

Wah kisin (tortilla del kisin)

Kuxum lu'um (hongos de la tierra)

$P$ 'ook kisin (sombrero del kisin)

Muruch

P'ook kisin (sombrero del kisin)

Kuxum che'(hongo de árbol)

Bay och (bolsa del tlacuache)

$P$ 'ook kisin (sombrero del kisin)

P'ook kisin (sombrero del kisin)

Pim wah kisin (tortilla del kisin)

Ax (verruga)

Ax (verruga), Baak-el kisin (hueso del

kisin)

Wah kisin (tortilla del kisin)

Lo'ro su compañero

Sak lo'ro (lo'ro blanco)

Bay och (bolsa de tlacuache)

Kuxum che'(hongo de árbol)

Wah kisin (tortilla del kisin)

Wah kisin (tortilla del kisin)

Leok, kuxum che' (hongo de árbol)

Leok, Chäk chaach su compañero

Kuxum che' (hongo de árbol)

$\mathrm{Su} \quad \mathrm{Li}$

$\mathrm{Su} \quad \mathrm{Li}$

$\mathrm{Su} \quad \mathrm{Te}$

$\mathrm{Su} \quad \mathrm{Te}$

$\mathrm{Su} \quad \mathrm{Te}$

$\mathrm{Su} \quad \mathrm{Te}$

$\mathrm{Su} \quad \mathrm{Te}$

$\mathrm{Su} \quad \mathrm{Li}$

$\mathrm{Su} \quad \mathrm{Li}$

$\mathrm{Su} \quad \mathrm{Li}$

$\mathrm{Su} \quad \mathrm{Li}$

$\mathrm{Su} \quad \mathrm{Li}$

$\mathrm{Su} \quad \mathrm{Hu}$

$\mathrm{Su} \quad \mathrm{Hu}$

$\mathrm{su} \quad \mathrm{Hu}$

$\mathrm{Me} \quad \mathrm{Te}$

$\mathrm{Me} \quad \mathrm{Te}$

$\mathrm{Su} \quad \mathrm{Te}$

$\mathrm{Su} \quad \mathrm{Te}$

$\mathrm{Su} \quad \mathrm{Li}$

$\mathrm{Su} \quad \mathrm{Li}$

$\mathrm{Su} \quad \mathrm{Hu}$

$\mathrm{Su} \quad \mathrm{Te}$

$\mathrm{Su} \quad \mathrm{Li}$

$\mathrm{Su} \quad \mathrm{Te}$

$\mathrm{Su} \quad \mathrm{Hu}$

$\mathrm{Co} \quad \mathrm{Hu}$

$\mathrm{Su} \quad \mathrm{Li}$

$\mathrm{Su} \quad \mathrm{Li}$

Co $\mathrm{Li}$

$\mathrm{Su} \quad \mathrm{Te}$

$\mathrm{Su} \quad \mathrm{Li}$

$\mathrm{Su} \quad \mathrm{Li}$

Me Te

$\mathrm{Me} \quad \mathrm{Te}$

$\mathrm{Su} \quad \mathrm{Li}$

Co $\quad \mathrm{Li}$

Co $\quad \mathrm{Li}$

$\mathrm{Lu} \quad \mathrm{Te}$

$\mathrm{Su} \quad \mathrm{Li}$

$\mathrm{Su} \quad \mathrm{Li}$

$\mathrm{Su} \quad \mathrm{Hu}$

Me, Li

Me, $\quad$ Li

$\begin{array}{ll}\mathrm{Ti} & \mathrm{Li} \\ \mathrm{Su} & \mathrm{Li}\end{array}$ 
Phylloporia spathulata (Hook.) Ryvarden Piptoporellus soloniensis (Dubois) B.K. Cui, M.L. Han \& Y.C. Dai, in Han, Chen, Shen, Song, Vlasák, Dai \&

Cui

Pleurotus djamor (Rumph. ex Fr.) Boedijn

Pluteus albostipitatus (Dennis) Singer

Pluteus sp.

Polyporus dictyopus Mont.

Polyporus leprieurii Mont.

Protohydnum cartilagineum Möller

Psathyrella sp.

Pseudofavolus tenuis (Fr.) G. Cunn.

Pseudohydnum gelatinosum (Scop.) P. Karst.

Psathyrella sp.

Pseudofavolus tenuis (Fr.) G. Cunn.

Pseudohydnum gelatinosum (Scop.) P. Karst.

Pterula plumosoides Corner

Pterula verticillata Corner

Pycnoporus sanguineus (L.) Murrill

Ramaria aff. albocinerea (Pat.) Corner

Ramaria grandis $f$. grandis (Peck) Corner

Ramaria subgen. Lentoramaria sp.

Ramariopsis sp.

Rhodoarrhenia sp.

Rhodofomitopsis feei (Fr.) B.K. Cui, M.L. Han \& Y.C.

Dai, in Han, Chen, Shen, Song, Vlasák, Dai \& Cui

Russula aff. mexicana Burl.

Russula mexicana Burl.

Schizophyllum commune Fr.

Scytinopogon $\mathrm{sp.}$

Suillus tomentosus (Kauffman) Singer

Teloschistes sp.

Tetrapyrgos nigripes (Fr.) E. Horak

Trametes elegans (Spreng.) Fr.

Trametes sp.

Trametes versicolor (L.) Lloyd

Trametes villosa (Sw.) Kreisel

Tremella sp.

Trichaptum perrottetii (Lév.) Ryvarden

Tricholomopsis sp.

Tricholosporum tropicale Guzmán, Bandala \& Montoya

Ustilago maydis (DC.) Corda

Xylaria anisopleura (Mont.) Fr.

Xylaria fockei (Miq.) Cooke

Xylaria longipes Nitschke

Xylaria multiplex (Kunze) Fr.

Xylaria obovata (Berk.) Berk.

Xylaria polymorpha (Pers.) Grev.
Kip sup, toop' $\quad \mathrm{Su}$

$\mathrm{Li}$

Wah kisin (tortilla del kisin)

$\mathrm{Su}$

$\mathrm{Li}$

Kayach, Kayoch

Co

$\mathrm{Li}$

Kibrum

Much'

Kuxum che' (hongo de árbol)

Misib kisin (escoba del kisin)

U ta'kisin (excremento del Kisin)

Chan ok (puñados pequeños)

Kuxum che' (hongo de árbol)

Yak' (lengua)

Chan ok (puñados pequeños)

Kuxum che' (hongo de árbol)

Yak' (lengua)

Misib kisin (escoba del kisin)

Yerek toop' kisin (flores del kisin)

Chäk wah kisin (tortilla roja del kisin)

Baak-el kisin (hueso del kisin)

U'jo kisin

Baak-el kisin (hueso del kisin)

Yerek toop' kisin (flores del kisin)

Lo'ro su compañero

Wah kisin (tortilla del kisin)

Chäk wah (tortilla roja)

Yoch wah

Xikin (oreja)

Misib kisin (escoba del kisin)

Wah ch'o' (tortilla del ratón)

Kuxum che' (hongo de árbol)

Toop 'che' (flores de árbol))

Xamuch kisin, Wah kisin (tortilla del

kisin)

Wah kisin (tortilla del kisin)

Wah kisin (tortilla del kisin)

Wah kisin (tortilla del kisin)

Lo'ro su compañero

Kuxum che' (hongo de árbol)

Kayoch su compañero

Yax p'ook kisin (sombrero verde del

kisin)

Ta'urim nar (hongo del maíz)

Baak-el kisin (hueso del kisin)

Baak-el kisin (hueso del kisin)

Halal kisin (flecha del kisin)

Kuxum che' (hongo de árbol)

Kip sup su compañero

Baak-el kisin (hueso del kisin)
$\mathrm{Ve} \quad \mathrm{Li}$

Co Li

$\mathrm{Su} \quad \mathrm{Li}$

$\mathrm{Su} \quad \mathrm{Li}$

su $\quad \mathrm{Li}$

$\mathrm{Su} \quad \mathrm{Hu}$

$\mathrm{Su} \quad \mathrm{Li}$

$\mathrm{Su} \quad \mathrm{Li}$

$\mathrm{Su} \quad \mathrm{Hu}$

$\mathrm{Su} \quad \mathrm{Li}$

$\mathrm{Su} \quad \mathrm{Li}$

$\mathrm{Me} \quad \mathrm{Li}$

$\mathrm{Me} \quad \mathrm{Li}$

$\mathrm{Me} \quad \mathrm{Li}$

$\mathrm{Su} \quad \mathrm{Li}$

$\mathrm{Su} \quad \mathrm{Te}$

$\mathrm{Su} \quad \mathrm{Li}$

Me Te

$\mathrm{Su} \quad \mathrm{Li}$

$\mathrm{Su} \quad \mathrm{Li}$

Ve Te

$\mathrm{Su} \quad \mathrm{Te}$

Co $\quad \mathrm{Li}$

$\mathrm{Su} \quad \mathrm{Te}$

$\mathrm{Su} \quad \mathrm{Te}$

$\mathrm{Su} \quad \mathrm{Li}$

$\mathrm{Su} \quad \mathrm{Li}$

$\mathrm{Su} \quad \mathrm{Li}$

$\mathrm{Su} \quad \mathrm{Li}$

$\mathrm{Su} \quad \mathrm{Li}$

$\mathrm{Su} \quad \mathrm{Li}$

$\mathrm{Su} \quad \mathrm{Li}$

$\mathrm{Su} \quad \mathrm{Li}$

Co $\quad \mathrm{Li}$

$\mathrm{Ve} \quad \mathrm{Hu}$

Co $\quad \mathrm{Pl}$

su $\quad \mathrm{Li}$

$\mathrm{Su} \quad \mathrm{Li}$

$\mathrm{Su} \quad \mathrm{Li}$

$\mathrm{Su} \quad \mathrm{Li}$

$\mathrm{Su} \quad \mathrm{Li}$

$\mathrm{Su} \quad \mathrm{Li}$ 


\begin{tabular}{|c|c|c|c|}
\hline Xylaria sp.1 & Baak-el kisin (hueso del kisin) & $\mathrm{Su}$ & $\mathrm{Li}$ \\
\hline Xylaria sp.2 & Kuxum tz'ib (escritura del kisin) & Ve & $\mathrm{Li}$ \\
\hline Xylaria sp. 3 & Baak-el kisin (hueso del kisin) & $\mathrm{Su}$ & $\mathrm{Li}$ \\
\hline Xylosphaera aff. comosa (Mont.) Dennis & $\begin{array}{l}\text { O'och urum kisin (alimento de guajolte } \\
\text { del kisin) }\end{array}$ & $\mathrm{Su}$ & $\mathrm{Li}$ \\
\hline Xylosphaera comosa (Mont.) Dennis & $\begin{array}{l}\text { O'och urum kisin (alimento de guajolte } \\
\text { del kisin), misib kisin (escoba del kisin) }\end{array}$ & $\mathrm{Su}$ & $\mathrm{Li}$ \\
\hline Xylosphaera phyllocharis (Mont.) Dennis & Kuxum tsip & $\mathrm{Su}$ & $\mathrm{Li}$ \\
\hline
\end{tabular}

Usos de los hongos

De las 164 especies reconocidas por los lacandones, 37 de ellas tienen algún uso, esto es el $23 \%$ del total. De estas, 18 son comestibles, 13 medicinales, 5 lúdicas, 2 tintóreas, una para embriagarse y una como agorera (Tabla 1).

Con relación a las especies comestibles, el 61\% son de sustrato lignícola y de una consistencia preferentemente correosa. Este fenómeno no es aislado sino más bien generalizado en la región tropical de México y del mundo. Autores como Prance (1984), Chacón (1988), Härkönen y colaboradores (1993), van Dijk y colaboradores (2003), Ruan-Soto y colaboradores (2006) y Vasco-Palacios y colaboradores (2008), señalan que los hongos consumidos en zonas tropicales son preferentemente de sustrato lignícola. Sin embargo, en Nahá, las personas entrevistadas también consumen algunas especies terrícolas como Agaricus spp. y Amanita complex. caesarea. Un aspecto a resaltar es que, pese a que en Nahá se distribuyen especies micorrizógenas altamente apreciadas como alimentos en todo el mundo como son Lactarius indigo o Suillus tomentosus, los entrevistados no los consideran como especies comestibles.

Los lacandones regularmente aprovechan las especies comestibles cuando se encuentran en los senderos por donde transitan en la selva y por supuesto en las milpas. Es en estos espacios donde, debido a las prácticas agrícolas tradicionales, existe una gran cantidad de materia leñosa producto de los árboles que son derribados para el establecimiento de los cultivos.
Yo voy con mi familia a ver la milpa, cada uno se hace cargo de algo, mi hijo y yo recogemos la mazorca y mis hijas y mi mujer acompañan recogiendo kayoch, ya en otra ocasión las mujeres ven el frijol y nosotros los hombres doblamos la milpa y recogemos el xikin (K, García, comunicación personal, Nahá, agosto de 2007).

Esta dinámica de recolecta es muy común entre los diferentes grupos humanos habitantes de las tierras bajas de Chiapas, como ya se ha documentado por Ruan-Soto, Cifuentes y colaboradores (2020), donde la milpa y los senderos son los principales espacios donadores de hongos comestibles y la recolecta se hace de manera oportunista.

Dentro de las especies consideradas como medicinales, existen aquellas que son utilizadas para ayudar a cicatrizar heridas de manera más eficiente al mismo tiempo de prevenir infecciones como el caso del Chäk wah kisin 'tortilla roja del kisin' (Pycnoporus sanguineus), o inclusive para eliminar verrugas de la piel utilizando a especies como Phaeoclavulina cokeri y P. zippelii, llamadas localmente ax que significa 'verruga'. Ambos taxones se colocan en el área afectada y son sujetados con un pedazo de tela o una venda esperando su efecto.

Dos especies del género Phillipsia, reconocidos localmente como leok, son utilizados como colorante para teñir fibras. Sin embargo, los propios entrevistados señalan que este conocimiento casi está desaparecido ya que los colorantes industriales han desplazado casi por completo las técnicas tradicionales. Este fenómeno de desplazamiento cultural y la consecuente 
pérdida de la vigencia de estas técnicas ancestrales de tinción son fenómenos comunes en todo el mundo (Maxia et al., 2013; Prigioniero et al., 2020).

\section{Importancia cultural de los hongos}

Los etnotaxones de mayor importancia cultural en las tres comunidades son el kayoch (Pleurotus dajmor y Favolus tenuiculus) con 30 menciones (88\% de la población), el xikin (Schizophyllum commune) con 28 menciones ( $82 \%$ de la población), el lo'ro (Auricularia spp.) con 25 menciones (73\%) el much (Agaricus spp.) con 9 menciones (26\%) y el bay och (Oudemansiella canarii con 8 menciones (23\%). Los tres primeros taxones son quizá los de mayor importancia cultural ya que se ha reportado su consumo en prácticamente todos los trabajos etnomicológicos realizados en los trópicos mesoamericanos y amazónicos (Chacón, 1988; Prance, 1984; Ruan-Soto et al., 2006; Vasco-Palacios et al., 2008).

Aunque estas especies aparentemente sean las más importantes entre los lacandones, el nivel de importancia es diferente entre comunidades. La Figura 2 muestra el análisis de clasificación basado en la frecuencia de mención relativa de hongos muestra un patrón de variación entre las tres comunidades. Un grupo reúne a Nahá y Metzabok, dejando a Lacanjá-Chansayab aparte con mayor diferencia. El análisis de componentes principales muestra que el componente principal 1 que explica el $83.22 \%$ de la variación discrimina a Nahá y Metzabok de Lacanjá-Chansayab (Figura 3). Los caracteres de mayor peso son la frecuencia de mención de los taxones Agaricus spp. y Ustilago maydis.

Como ya se ha mencionado, existen variaciones documentadas entre los lacandones del sur (Lacanjá-Chansayab) y del norte (Nahá y Metzabok), producto quizá de su origen histórico distinto. Estas diferencias se observan en algunas prácticas, formas de organización social y principalmente en su lengua. $\mathrm{Si}$ bien los resultados aquí presentados son obtenidos a partir de una muestra pequeña y por tanto podrían ser no concluyentes, sí muestran una tendencia que potencialmente refleja que las diferencias culturales entre los lacandones del norte y del sur también incluyen el conocimiento acerca de los hongos silvestres. Ejemplo de esto es el conocimiento acerca de Ustilago maydis entre los lacandones del sur y el consumo casi exclusivo de diferentes especies del género Agaricus por los lacandones septentrionales.

\section{Figura 2}

Análisis de clasificación basado en la frecuencia de mención relativa de hongos.

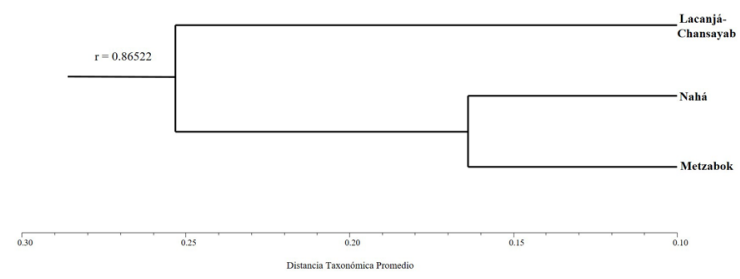

Figura 3

Análisis de Componentes Principales basado en la frecuencia de mención relativa de hongos.

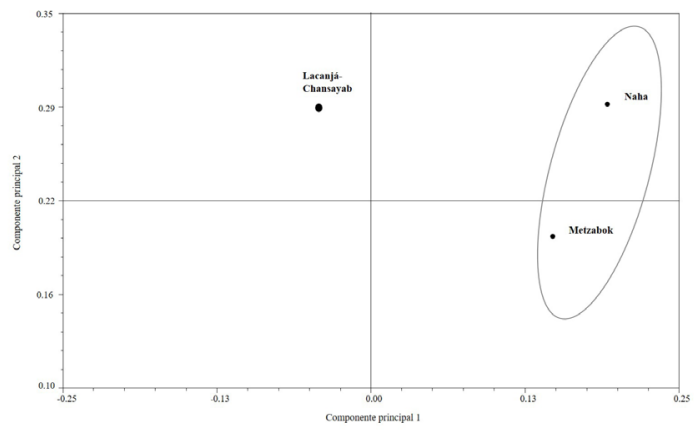

Reflexiones finales

Resulta extraño que antes del 2005, en casi 100 años de estudios sobre los lacandones, no se hayan documentado aspectos relacionados con el conocimiento y uso de los hongos por este grupo originario en la selva chiapaneca. Esto es quizá reflejo de la inherente falta de estudios de los hongos en general en estos ambientes tropicales (Guzmán, 2003). Por mucho tiempo, el estudio de los hongos en los trópicos estuvo muy poco desarrollado, dándose prioridad a la revisión de especies propias de latitudes boreales y de altitudes mayores a $1500 \mathrm{~m}$ s.n.m.

Sin embargo, el reconocimiento de 164 especies es de resaltar entre los grupos mayas estudiados hasta el momento en todo el estado de Chiapas. Por lo general, no existe reporte de un número tan alto de especies importantes culturalmente en lo reportado hasta el momento para grupos mayas en particular y/o habitantes 
de tierras bajas tropicales mesoamericanas en general (Ruan-Soto \& Ordaz Velázquez, 2015).

Es de llamar la atención que, del total de especies conocidas, solamente 37 de ellas tienen algún uso, número menor si se compara con otros grupos mayas de los Altos de Chiapas (Ruan-Soto, 2018b; Ruan-Soto, Cifuentes et al., 2020). En la etnobiología son conocidas dos propuestas teóricas al respecto de la motivación para asignar un nombre a las especies y no solamente caracterizarlas con denominadores genéricos: la propuesta cognitivista y la pragmática. Para Berlin (1992) el objetivo principal al clasificar no es práctico, sino que implica requerimientos intelectuales que van más allá de las necesidades pragmáticas, es decir, existe una necesidad intelectual de clasificar el mundo y los elementos que contiene. La categorización de taxones está basada en afinidades y diferencias morfológicas, independientemente de su importancia cultural. Por otro lado, autores como Bulmer (1967), Douglas (1998) o Hunn (1982), los principios en que se basan las clasificaciones humanas del mundo están centrados en su grado de proximidad del dominio humano. En este sentido, cada taxón que recibe un nombre lo hace ya sea porque ha sido usado de alguna manera o porque goza de cierto interés para el grupo humano en cuestión (Levi-Strauss, 1964). En este sentido, aunque solamente el $22 \%$ de las especies que los lacandones nombran y clasifican tienen un uso, el $78 \%$ restante no es carente de interés. Como puede verse a partir de las narrativas, muchas de las especies recrean en sus nombres y clasificación mitos de origen de los hongos y la relación que guardan estos organismos con los dioses del panteón lacandón.

Resalta la identidad de Pleurotus dajmor, Favolus tenuiculus, Schizophyllum commune y Auricularia spp. como los taxones comestibles de mayor importancia, patrón que se repite en otras comunidades estudiadas de tierras bajas mesoamericanos e incluso latinoamericanas.

Si bien los resultados de este estudio no pueden considerarse como concluyentes en su parte cuantitativa, aparentemente muestran que al menos una parte de los conocimientos etnomicológicos, quizá más ligada con las prácticas de aprovechamiento de las especies comestibles, pueden ser diferentes entre los lacandones del norte y los del sur, reflejando posiblemente tradiciones históricas de mayor duración al tiempo de homogenización planteada por algunos investigadores.

Estudios como estos contribuyen a conocer un poco más de los recursos fúngicos de regiones poco estudiadas como el sureste mexicano, así como a do- cumentar aspectos del conocimiento etnomicológico local. A través de esto es posible caracterizar de manera más completa otros aspectos del patrimonio biocultural mesoamericano.

\section{Agradecimientos}

Se agradece a la Comisión Nacional de Áreas Naturales Protegidas, particularmente al Área de Protección de Flora y Fauna Naha y Metzabok, el apoyo para el desarrollo de esta investigación. Este trabajo fue apoyado parcialmente por el Consejo Nacional de Ciencia y Tecnología de México a través de la beca No. 23444 al primer autor. Se agradece a Dykarion Language Consultants la traducción del resumen y la revisión de estilo.

\section{Referencias}

Baer, P., \& Merrifield, W. R. (1981). Los lacandones de México: Dos estudios. Instituto Nacional Indigenista.

Berlin, B. (1992). Ethnobiological classification: Principles of categorization of plants and animal in traditional societies. Princeton University Press.

Bernard, R. (2006). Research methods in anthropology. Altamira Press.

Boremanse, D. (1978). The social organization of the lacandon indians of Mexico [Tesis de doctorado no publicada]. Oxford University.

Bruce, R. (1976). Textos y dibujos lacandones de Nahá. Instituto Nacional de Antropología e Historia.

Bruce, R., Robles, U., \& Ramos, E. (1971). Los lacandones: Cosmovisión maya. Departamento de investigaciones antropológicas del Instituto $\mathrm{Na}-$ cional de Antropología e Historia.

Bulmer, R. (1967). Why the Cassorwary in Not a bird. Man, 2(1), 5-25.

Cano-Contreras, E., Erosa Solana, E., \& Mariaca, R. (2009). Tu chien k'an: Un recorrido por la cosmovisión de los lacandones del Norte desde las mordeduras de serpiente. Universidad Intercultural de Chiapas, Sociedad Latinoamericana de Etnobiología, El Colegio de la Frontera Sur. 
Cano-Contreras, E., Medinaceli, A., Sanabria Diago, O. L., Argueta Villamar, A. (2015). Código de ética para la investigación, la investigación-acción y la colaboración etnocientífica en América Latina. Versión Dos. Etnobiología, 13(4), 3-22.

Cardoso, D., Queiroz, L., Bandeira, F., \& Góes-Neto, A. (2010). Correlations between indigenous brazilian folk classifications of fungi and their systematics. Journal of Ethnobiology, 30(2), 252-264.

Castillo-Campos, G., \& Nareve, H. (1992). Contribución al conocimiento de la vegetación de la Reserva de la Biosfera Montes Azules, Selva Lacandona, Chiapas, México. En M. A. Vázquez-Sánchez y M. A. Ramos (Eds.), Reserva de la Biosfera Montes Azules, Selva Lacandona: Investigación para su conservación (pp. 51-85.). Centro de Estudios para la Conservación de los Recursos Naturales.

Chacón, S. (1988). Conocimiento etnoecológico de los hongos en Plan de Palmar, Municipio de Papantla, Veracruz, México. Micología Neotropical Aplicada, 1, 45-54.

Chang, Y. S., \& Lee, S. S. (2004). Utilization of macrofungi species in Malaysia. Fungal Diversity, $15,15-22$.

Cifuentes, J., Villegas, M., \& Pérez-Ramírez, L. (1986). Hongos. En A. Lot \& F. Chang (Eds.), Manual de herbario (pp. 55-64.). Consejo Nacional de la Flora de México.

Codija, J. E. I., \& Yororu, N. A. (2014). Ethnicity and gender variability in the diversity, recognition and exploitation of Wild Useful Fungi in Pobè region (Benin, West Africa). Journal of Applied Biosciences, 78, 6729-742. https://doi.org/10.4314/ jab.v78i1.14

Comandini, O., \& Rinaldi, A. (2020). Ethnomycology in Europe: The Past, the Present, and the Future. En J. Perez-Moreno, A. Guerin-Laguette, R. Flores Arzu \& F. Q. Yu (Eds.), Mushrooms Humans and Nature in a Changing World (pp. 341-364). Springer.

Comisión Nacional de Áreas Naturales Protegidas. (2006a). Programa de conservación y manejo Área de Protección de Flora y Fauna Metzabok.

Comisión Nacional de Áreas Naturales Protegidas. (2006b). Programa de conservación y manejo Área de Protección de Flora y Fauna Nahá.
Contreras, U., \& Mariaca, R. (2016). Manejo de los recursos naturales entre los mayas lacandones de Nahá. El Colegio de la Frontera Sur, Red Temática sobre el Patrimonio Biocultural.

Cook, S. (2016). The Forest of the Lacandon Maya: An Ethnobotanical Guide. Springer.

De Vos, J. (1980). La paz de Dios y del Rey. La conquista de la Selva Lacandona. Fondo de Cultura Económica.

Douglas, M. (1998). Estilos de pensar. Gedisa.

Durán, A. (1999). Estructura y etnobotánica de la Selva alta perennifolia de Nahá, Chiapas (Tesis de maestría no publicada). Universidad Nacional Autónoma de México.

Durand, L., Figueroa, F., \& Trench, T. (2014). Inclusion and Exclusion in Participation Strategies in the Montes Azules Biosphere Reserve, Chiapas, Mexico. Conservation \& Society, 12(2), 175-189.

England, N. (1996). Introducción a la lingüística idiomas mayas. Cholsamaj.

Erosa, E. (2006). Lacandones. Comisión Nacional para el Desarrollo de los Pueblos Indígenas.

García-Gil, J. G., \& Lugo, J. (1992). Las formas del relieve y los tipos de vegetación en la selva lacandona. En M. A. Vázquez-Sánchez \& M. A. Ramos (Eds.), Reserva de la Biosfera Montes Azules, Selva Lacandona: Investigación para su conservación (pp. 51-85). Centro de Estudios para la Conservación de los Recursos Naturales.

Garibay-Orijel, R., \& García-Medrano, S. (2006). Importancia ecológica de los hongos. En J. Tovar-Velasco \& R. Valenzuela (Eds.), Los hongos del Parque Nacional Desierto de los Leones (pp. 101-108). Gobierno del Distrito Federal, Secretaría del Medio Ambiente.

Garibay-Orijel, R., Caballero, J., Estrada-Torres, A., \& Cifuentes, J. (2007). Understanding cultural significance, the edible mushrooms case. Journal of Ethnobiology and Ethnomedicine, 3, 4.

Goés-Neto, A., \& Bandeira, F. P. (2003). A Review of the Ethnomycology of Indigenous People in Brazil and its Relevance to Ethnomycologycal Investigation in Latin America. Revista Mexicana de Micología, 17, 11-16.

Guzmán, G. (1987). Distribución y etnomicología de Pseudofistulina radicata en Mesoamérica, con 
nuevas localidades en México y su primer registro en Guatemala. Revista Mexicana de Micologia, (3), 29-38.

Guzmán, G. (2003). Los hongos del Edén Quintana Roo. Introducción a la micobiota tropical de México. Instituto de Ecología.

Guzmán-Dávalos, L., \& Guzmán, G. (1979). Estudio ecológico comparativo entre los hongos (macromicetos) de los bosques tropicales y los de coníferas del sureste de México. Boletín de la Sociedad Mexicana de Micología, 13, 89-125.

Härkönen, M., Saarimäki, T., Mwasumbi, L., \& Niemelä, T. (1993). Collection of the tanzanian mushroom heritage as a form of developmental cooperation between the univertsities of Helsinki and Dar es Salaam. Aquilo Seria Botanica, 31, 99-105.

Hawksworth, D. L., \& R. Lücking (2017). Fungal Diversity Revisited: 2.2 to 3.8 Million Species. Mycrobiology Spectrum, 5(4). https://doi. org/10.1128/microbiolspec.FUNK-0052-2016.

Hernández-Sánchez, M. (2007). Percepción de los cambios en la vegetación y de los hongos macroscópicos en la región del Usumacinta, Tabasco (Tesis de licenciatura no publicada). Universidad Juárez Autónoma de Tabasco.

Hunn, E. (1982). The utilitarian factor in folk biological classification. American Anthropologist, 84(4), 830-847. https://doi.org/10.1525/ aa.1982.84.4.02a00070

Instituto Nacional de Ecología. (2000). Programa de manejo de la Reserva de la Biosfera Montes Azules. Ciudad de México: Instituto Nacional de Ecología.

Instituto Nacional de Estadística y Geografía. (2010). Censo general de población y vivienda. https:// www.inegi.org.mx/programas/ccpv/2010/

Jiménez, J. (2017). Etnomicología en la región náhuatl de San Luis Potosí: importancia cultural y propiedades bioquímicas (Tesis de maestría no publicada). Universidad Autónoma de Querétaro.

Kashanipour, R. A., \& Mcgee, R. J. (2004). Northern lacandon maya medicinal plant use in the communities of Lacanja Chan Sayab and Naha', Chiapas, Mexico. Journal of Ecological Anthropology, $8(1), 47-66$.
Largent, D., Johnson, D., \& Watling, R. (1977). How to identify mushrooms to genus. III: Microscopic features. Mad River.

Levi-Strauss, C. (1964). El pensamiento salvaje. Fondo de Cultura Económica.

Levy-Tacher, S. (2000). Sucesión causada por la roza - tumba - quema en las selvas de Lacanhá, Chiapas (Tesis de doctorado no publicada). Colegio de Postgraduados, Texcoco.

Levy-Tacher, S. I., Aguirre-Rivera, J. R., Martínez-Romero, M. M., \& Durán-Fernández, A. (2002). Caracterización del uso tradicional de la flora espontánea en la comunidad lacandona de Lacanhá. Interciencia, 27(10), 512-520.

López Austin, A. (1990). Los mitos del tlacuache. Instituto de Investigaciones Antropológicas de la Universidad Nacional Autónoma de México.

Mapes, C., Bandeira, F., Caballero, J., Goés-Neto, A. (2002). Mycophobic or Mycophilic? a comparative ethnomycological study between Amazonia and Mesoamerica. En R. Stepp, F. Wyndham \& R. Zarger (Eds.), Ethnobiology and biocultural diversity. Proceedings of the Seventh International Congress of Ethnobiology (pp. 180-188). University of Georgia Press.

Mapes, C., Guzmán, G., \& Caballero, J. (1981). Etnomicología Purépecha. El conocimiento y uso de los hongos en la cuenca del lago de Pátzcuaro, Michoacán (Serie Etnociencias 2). Dirección General de Culturas Populares, Secretaría de Educación Pública, Sociedad Mexicana de Micología, Instituto de Biología de la Universidad Nacional Autónoma de México.

March, I. (1998). Los mayas lacandones. Lacandones: Problemas y potencialidades para desarrollo de un grupo indígena minoritario. El Colegio de la Frontera Sur.

Marion Singer, M.-O. (1999). El poder de las hijas de la luna. Consejo Nacional para la Cultura y Las Artes y Plaza y Valdez.

Marion Singer, M.-O. (2000). Bajo la sombra de la gran ceiba: La cosmovisión de los lacandones. Desacatos, (5), 45-56.

Maxia, A., Meli F., Gaviano, C., Picciau, R., de Martis, B., Kasture, S., \& Kasture, V. (2013). Dye Plants: Natural resources from traditional bota- 
nical knowledge of Sardinia Island, Italy. Indian Journal of Traditional Knowledge, 12(4), 651656.

Miranda, F. (1952). La vegetación de Chiapas. Ediciones del Gobierno del Estado.

Montoya, A., Torres-García, E., Kong, A., Estrada-Torres, A., \& Caballero, J. (2012). Gender differences and regionalization of the cultural significance of wild mushrooms around La Malinche volcano, Tlaxcala, México. Mycologia, 104(4), 826-834. https://doi.org/10.3852/11-347

Moreno-Fuentes, A., Garibay-Orijel, R., Tovar-Velasco, J., \& Cifuentes, J. (2001). Situación actual de la Etnomicología en México y el mundo. Etnobiología, (1), 75-84.

Moreno-Fuentes, A., \& Garibay-Orijel, R. (2014). La Etnomicología en México. Estado del Arte. Red de Etnoecología y Patrimonio Biocultural, Universidad Autónoma del Estado de Hidalgo, Instituto de Biología, Sociedad Mexicana de Micología, Asociación Etnobiológica Mexicana, Grupo Interdisciplinario para el Desarrollo de la Etnomicología en México, Sociedad Latinoamericana de Etnobiología.

Nations, J. D., \& Nigh, R. B. (1980). The evolutionary potential of lacandon maya sustained-yield tropical rain forest agriculture. Journal of Anthropological Research, 36(1), 1-33.

Pennington, T. D., \& Sarukhan, J. (1998). Árboles tropicales de México. Ciudad de México: Universidad Nacional Autónoma de México - Fondo de Cultura Económica.

Prance, G. T. (1984). The use of edible fungi by amazonian indians. En G. T. Prance \& M. Kallunki (Eds.), Ethnobotany in the neotropics. NY Botanical Garden Publication (Vol. 1). Allen Press.

Prigioniero, A., Geraci, A., Schicchi, R., Tartaglia, M., Zuzolo, D., Scarano, P., Marziano, M., Postiglione, A., Sciarrillo, R., \& Guarino, C. (2020). Ethnobotany of dye plants in Southern Italy, Mediterranean Basin: floristic catalog and two centuries of analysis of traditional botanical knowledge heritage. Journal of Ethnobiology and Ethnomedicine, 16, Article 31. https://doi.org/10.1186/ s13002-020-00384-2

Quintana-Ascencio, P., Ramírez-Marcial, N., \& González-Espinosa, M. (1990). El medio natural de la región de Bonampak, Selva Lacandona, Chiapas. Centro de Investigaciones Ecológicas del Sureste.

Roblero, M. (2008). La relación hombre-naturaleza entre los lacandones de Nahá, Ocosingo, Chiapas. Liminar, 6(1), 125-140.

Rohlf, F. J. (2005). NTSYS-pc: Numerical Taxonomy and Multivariate Analysis System (Version 2.2) [Software].

Ruan-Soto, F. (2018a). Sociodemographic differences in the cultural significance of edible and toxic mushrooms among Tsotsil towns in the Highlands of Chiapas, Mexico. Journal of Ethnobiology and Ethnomedicine, 14(1), 32. https://doi. org/10.1186/s13002-018-0232-9

Ruan-Soto, F. (2018b). Recolección de hongos comestibles silvestres y estrategias para el reconocimiento de especies tóxicas entre los tsotsiles de Chamula, Chiapas, México. Scientia Fungorum, 48, 1-13. https://doi.org/10.33885/sf.2018.48.1179

Ruan-Soto, F. (2020). Highly cultural signicant edible and toxic mushrooms among the Tseltal from the Highlands of Chiapas, Mexico. Ethnobiology and Conservation, 9, 32. https://doi.org/10.15451/ ec2020-08-9.32-1-20

Ruan-Soto, F., Cifuentes, J., Garibay-Orijel, R., \& Caballero, J. (2020). Comparación de la disponibilidad de hongos comestibles en tierras altas y bajas de Chiapas, México, y sus implicaciones en estrategias tradicionales de aprovechamiento. Acta Botánica Mexicana, (128), Article el731. https:// doi.org/10.21829/abm128.2021.1731

Ruan-Soto, F., Cifuentes, J., Pérez-Ramírez, L., Ordaz-Velázquez, M., \& Caballero, J. (2021) Hongos macroscópicos de interés cultural en los Altos de Chiapas y la selva Lacandona, México. Revista Mexicana de Biodiversidad, 92, 1-24. https://doi. org/10.22201/ib.20078706e.2021.92.3525

Ruan-Soto, F., Garibay-Orijel, R., \& Cifuentes, J. (2004). Conocimiento Micológico Tradicional en la Planicie Costera del Golfo de México. Revista Mexicana de Micología, 19, 57-70.

Ruan-Soto, F., Garibay-Orijel, R., \& Cifuentes, J. (2006). Process and dynamics of traditional selling wild edible mushrooms in tropical Mexico. Journal of Ethnobiology and Ethnomedicine, 2, Article 3. https://doi.org/10.1186/1746-4269-2-3 
Ruan-Soto, F, Mariaca, R., Cifuentes, J., Limón, F., Pérez-Ramírez, L., \& Sierra-Galván, S. (2007). Nomenclatura, clasificación y percepciones locales acerca de los hongos en dos comunidades de la Selva Lacandona, Chiapas, México. Etnobiología, 5, 1-20.

Ruan-Soto, F., \& Ordaz-Velázquez, M. (2015). Aproximaciones a la etnomicología maya. Pueblos y Fronteras, 10(20), 44-69.

Ruan-Soto, F., Pérez Ramírez, L., Cifuentes Blanco, J., Ordaz-Velázquez, M., Cruz Solís, A., García del Valle, Y., Reyes Escutia, F., \& Mariaca Méndez, R. (2017). Hongos de los lacandones de Nahá y Metzabok: Guía ilustrada de macromicetos. El Colegio de la Frontera Sur.

Ruan-Soto, F., Ramírez-Terrazo, A., Montoya, A., \& Garibay-Orijel, R. (2020). Métodos en etnomicología. Instituto de Biología de la UNAM-Sociedad Mexicana de Micología.

Sandoval, C. (2002). Investigación cualitativa. Programa de especialización teórica, métodos y técnicas de investigación social. Instituto Colombiano para el Fomento de la Educación Superior.

Thomas, E., Vanderbroek, I., Van Damme, P. (2007). What works in the field? A comparison of different interviewing methods in ethnobotany with special reference to the use of photographs. Economic Botany, 61(4), 376-384. https://doi.org/1 0.1663/0013-0001(2007)61[376:WWITFA]2.0. $\mathrm{CO} ; 2$

Tozzer, A. M. (1982). Mayas y Lacandones, Un estudio comparativo. Instituto Nacional Indigenista.

Trench, T. (2005). Representaciones y sus impactos: El caso de los lacandones en la Selva Lacandona. Liminar, 3(2), 48-69. https://doi.org/10.29043/liminar.v3i2.182

Turner, N. J. (1988). The Importance of a rose: Evaluating the cultural significance of plants in Thompson and Lillooet interior Salish. American Anthropologist, 90(2), 272-290. https://doi.org/10.1525/ aa.1988.90.2.02a00020

van Dijk, H., Awana Onguene, N., \& Kuyper, T. W. (2003). Knowledge and utilization of edible mushrooms by local populations of the rain forest of south Cameroon. Ambio, 32(1), 19-23. https://doi. org/10.1579/0044-7447-32.1.19
Vasco-Palacios, A. M., Suaza, S. C., Castaño-Betancur, M., \& Franco-Molano, A. E. (2008). Conocimiento etnoecólogico de los hongos entre los indígenas Uitoto, Muinane y Andoke de la Amazonía Colombiana. Acta Amazónica, 38(1), 17-30. https:// doi.org/10.1590/S0044-59672008000100004

Villa-Rojas, A. (1985). Los mayas: Estudios etnológicos. Instituto de Investigaciones Antropológicas de la Universidad Nacional Autónoma de México.

Weller, S. C., Romney, A. K. (1988). Systematic data collection. Sage Publications.

Zent, E. L., Zent, S., \& Iturriaga, T. (2004). Knowledge and Use of Fungi by a Mycophilic Society of the Venezuelan Amazon. Economic Botany, 58(2), 214-226. https://doi.org/10.1663/0013-000 1(2004)058[0214:KAUOFB]2.0.CO;2 